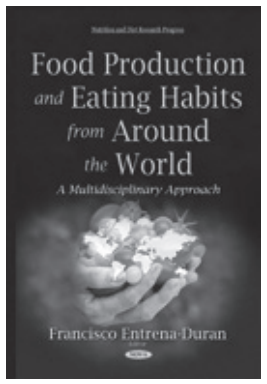

\title{
Francisco Entrena-Duran (editor). Food Production and Eating Habits From Around the World: A Multidisciplinary Approach.
}

\author{
Nueva York: Nova Publishers, 2015, 248 p. \\ ISBN: 978-1-63482-540-5
}

Simón Pedro Izcara Palacios ${ }^{1}$

\begin{abstract}
Actualmente nuestros comportamientos alimentarios siguen produciéndose en escenarios locales, pero, al mismo tiempo que estos escenarios van dejando atrás su tradicional localismo, se vinculan cada vez más a la globalización; es decir, experimentan una creciente glocalización. Esta se manifiesta en el doble sentido de localización de lo global y de globalización de lo local. Por consiguiente, la glocalización implica procesos de indigenización de la modernidad urbano-industrial dominante a escala global (Sahlins, 2000), así como de modernización de las tradiciones locales. En esta situación, a la vez que cobran fuerza tendencias hacia la homogeneización y/o uniformización de los hábitos alimenticios alrededor de todo el planeta $^{2}$, las peculiaridades alimentarias que simbolizan los específicos entornos locales parecen difundirse más y más (Serra-Mallol, 2013). De este modo, la alimentación es a menudo el último marcador cultural en dejar vía libre a la influencia multidimensional del modelo cultural dominante (Goody, 1985; De Garine, 1996). Ello explicaría que, a pesar de los indudables efectos uniformadores de la globalización sobre los hábitos alimenticios mundiales, la comida siga desempeñando un destacado papel en la formación de las identidades sociales, si bien es verdad que esas identidades son cada vez más mestizas. Pero,
\end{abstract}

\footnotetext{
1 Universidad Autónoma de Tamaulipas (México). E-mail: sizcara@uat.edu.mx

2 Sobre todo, como consecuencia del influjo de los medios de comunicación y de las crecientes facilidades para el transporte y la distribución transnacional de los productos agrarios y/o alimenticios.
}

este mestizaje no hay que verlo solo como el fruto de una imposición de lo global, sino también, en muy gran medida, como resultado de elecciones de los sujetos más o menos libremente adoptadas o deseadas (Corbeau, 2008: 117-118).

En las presentes circunstancias de glocalización alimentaria el libro que les presento resulta muy oportuno. En él, su editor, el Catedrático de Sociología de la Universidad de Granada (España) Francisco Entrena-Duran, reúne una selección de trabajos escritos por especialistas en disciplinas tan diversas como la Antropología, la Comunicación, la Economía, la Enfermería, el Marketing, la Medicina, la Psicología o la Sociología. En esta diversidad de ubicaciones disciplinares de sus autores están las fortalezas y las debilidades del libro. Particularmente, en cuanto a las segundas, no espere el lector encontrar aquí una obra que parte de un enfoque teórico-metodológico preciso y luego sigue un único y coherente hilo argumental en consonancia con ello, sino que, por el contrario, en cada uno de los diferentes capítulos del libro se afronta el problema estudiado desde unas perspectivas en consonancia con la referida diversidad de disciplinas en las que laboran sus respectivos autores.

Desde luego, sí estamos ante un volumen de naturaleza y alcance globales. Sus autores trabajan en universidades o instituciones de investigación de los 5 continentes del mundo, y los asuntos que en él se tratan solo pueden ser adecuadamente entendidos si se los sitúa en el marco de la globalización. El propio editor, que también es autor de 3 de los 14 
capítulos del libro, es plenamente consciente de esto, tal y como da a entender el título Globalization as the Context to Understand Changes in Food Production and Eating Habits que pone a su prefacio introductorio.

En particular, en lo que a Food Production (Parte 1) se refiere, el libro muestra cómo actualmente, cuando muchas sociedades rurales contemporáneas están experimentando procesos de desagrarización, tiene lugar una progresiva industrialización e intensificación de los procesos de producción de comida. Los costes ambientales y económicos, así como los problemas que plantea la agricultura industrial, son considerablemente mayores que los de la agricultura ecológica sostenible. Sobre todo, porque los precios a los que se venden los alimentos industriales externalizan los costes de los problemas ambientales que generan (p. 5).

Para quien escribe esta reseña, uno de tales problemas es la reducción de la biodiversidad. Las referencias a ella que se hacen en diferentes partes del libro resultan especialmente oportunas, ya que, en una situación de cambios climáticos como la actual, necesitamos de una fuerte biodiversidad en los territorios locales para que las sociedades de estos sean más resilientes en lo que respecta a su capacidad de adaptarse a dichos cambios. Ello significa que, sin caer en una especie de mitificación del aislamiento y la autarquía, haría falta preservar y promover los cultivos autóctonos, tender a producir en cada territorio la mayoría de lo que en él se consume. Por ejemplo, mediante el apoyo a los circuitos cortos de producción y comercialización, tal y como señala José Ramón Mauleon (2001). Un objetivo primordial es que la acción de producir alimentos contribuya a mantener vivos los territorios locales, a preservar y reproducir las comunidades que los sustentan (López García, 2015), al mismo tiempo que a asegurar la soberanía alimentaria dentro de ellos, lo cual, a su vez, es un prerrequisito esencial para garantizar su seguridad alimentaria (Esquinas Alcázar, 2013).

Como ya dijo el Presidente Kennedy hace más de 50 años, "en nuestro tiempo tenemos la capacidad para eliminar el hambre de la faz de la tierra. Necesitamos sólo la voluntad de hacerlo (1963)". No basta, pues, con que seamos capaces de producir suficientes alimentos para erradicar el hambre y asegurar el derecho a la alimentación ${ }^{3}$, ya que la suficiencia y/o la seguridad alimentaria no garantizan por sí solas la soberanía alimentaria, entendida esta como la capacidad de la mayoría de la población para acceder a los alimentos que necesita a unos precios asequibles y al margen de las operaciones financiero-especulativas y de los vaivenes inherentes a la volatilidad de los mercados (Sumpsi Viñas, 2013).

En este escenario, como se señala en el libro, una considerable parte de la población mundial no dispone de recursos económicos suficientes para procurarse una dieta suficiente y saludable. Además, muchos de los que sí cuentan con esos recursos, tampoco se alimentan adecuadamente, debido a que, por carencias educativas, no ven la necesidad de modificar sus hábitos de adquisición y de consumo de alimentos. Al no tener una conciencia clara acerca de su responsabilidad como consumidores, no saben lo que es una dieta saludable, ni tampoco la desean, aunque puedan necesitarla urgentemente. Ello, junto con la escasez de tiempo de muchas mujeres incorporadas al mercado laboral para atender las tareas que los estereotipos machistas siguen percibiendo como consustanciales a su condición de género, crea unas condiciones idóneas para el presente crecimiento de la comida rápida o basura. De ahí, el aumento de distintos problemas de salud, tales como la obesidad, la diabetes o los demasiado altos niveles de colesterol, etc. (p. 8 y siguientes).

Por otra parte, los procesos de cambio en los sistemas de producción, comercialización y consumo de la comida, así como los problemas y las mutaciones en los hábitos alimentarios analizados en este volumen, son contextualizados por el editor (Prefacio y capítulos 1 y 14) en el marco de las transformaciones tecnológicas y socioproductivas experimentadas como consecuencia del tránsito del mundo rural tradicional a las presentes sociedades predominantemente urbano-in-

3 Los lectores pueden encontrar diversos estudios acerca del derecho a la alimentación, realizados desde diferentes enfoques, en AA.VV (2006: 1-332), así como en AA.VV (2008: 1-450). 
dustriales, cada vez más globalizadas. Frente a las tendencias hacia la homogenización alimentaria mundial de tales sociedades, se experimentan, en la mayoría del planeta, reacciones de búsqueda de la distinción mediante el reforzamiento o la reconstrucción de las singularidades alimentarias locales. Esta situación explica que la búsqueda de la identidad, la especificidad o la distinción, a través de la adquisición y el consumo de alimentos, sean lugares comunes en bastantes de los capítulos compendiados en este libro, independientemente de la zona mundial de procedencia de sus respectivos autores.

También son lugares comunes las preocupaciones generalizadas de esos capítulos por la extensión de estilos de vida cada vez más sedentarios y de procedimientos de producción de comida industrial bastante opacos para el gran público, así como por los efectos negativos sobre la salud de los medios publicitarios y/o de comunicación. Estos, a menudo, inducen, desde la infancia (Véase la Parte IV: Children's Eating Habits) y a lo largo de las diferentes etapas de la vida, al desarrollo de formas inadecuadas de consumo alimentario, no solo en los casos en los que dan lugar a sobrepeso y obesidad, sino también cuando están alentadas por discursos que propugnan la adquisición de ideales de naturaleza o formas corporales y arquetipos de belleza tan poco saludables como prácticamente imposibles de conseguir, pero que suelen causar anorexia y bulimia (Véase la Parte II: Eating Habits, Physical Activity, Body and Health).

La salud no es solamente considerada en el libro desde el punto de vista de los médi$\cos$ y/o los nutricionistas (interesados, obviamente, en las amenazas que para ella pueden derivarse de la inadecuada alimentación), sino que también se nos muestra cómo las crecientes preocupaciones de la población por la salud Ilegan incluso a ser instrumentalizadas por los discursos de la publicidad con respecto a determinados productos alimentarios, cuyos supuestos beneficios para la salud constituyen en realidad reclamos para atraer la atención de los consumidores (Véase la Parte III: Advertising and Discourses on Food).

Estos y otros asuntos son tratados a lo largo de las diferentes partes de esta completa obra colectiva, la cual finaliza con la parte
IV, dedicada a Changes in Eating Habits. Tales hábitos son considerados como socialmente producidos (p. 237), a la vez que se muestra cómo sus procesos de construcción social, que están crecientemente determinados por el influjo de los medios de comunicación y la publicidad, tienden a dejar de desarrollarse prioritariamente en el seno de la institución familiar y a producirse de manera cada vez más individualizada.

Tanto por la variedad de temáticas que trata, como por la diversidad de perspectivas desde las que lo hace (interdisciplinariedad), la obra que aquí se reseña es de interés para científicos y profesionales sociales, publicistas, nutricionistas y todos aquellos, de una $u$ otra forma, implicados o interesados en las problemáticas de la producción de comida y/o de los hábitos alimentarios. Agrupa a especialistas de muy diferentes lugares del mundo y cuyos enfoques teórico-analíticos son significativamente diversos. Sin embargo, independientemente de sus variadas perspectivas y disciplinas científicas de trabajo, todos estos especialistas coinciden en que se ocupan de procesos y problemas alimentarios similares. En gran medida, esta coincidencia se debe a que, pese a la evidente heterogeneidad geopolítica y socioeconómica de los contextos en los que transcurren sus vidas y se desenvuelven sus actividades investigadoras y/o profesionales, los autores de los diferentes capítulos forman todos ellos parte de la cada vez más interconectada presente sociedad global. Se explica así que, más allá de sus especificidades locales, los problemas y los hábitos alimentarios de los que tratan los trabajos incluidos en este volumen tengan todos en común la circunstancia de estar crecientemente insertos en la globalización y afectados en sus consecuencias y dinámicas por ella; en definitiva, están cada vez más glocalizados. De ahí, la importancia y la oportunidad de acometer el análisis de la producción de comida y de los hábitos alimentarios mediante estudios multidisciplinares de alcance mundial como el de la experiencia de colaboración académica que ha conllevado la redacción del libro que aquí se presenta. Un libro que muy probablemente no pasará desapercibido, no solo por la calidad y el interés de los trabajos que su editor (autor de 128 trabajos -entre libros, capítulos de libro y artículos- aparecidos en editoriales 
y revistas de difusión internacional) ha compendiado, sino también porque los actuales problemas y cambios en la producción de comida y en la construcción de los hábitos alimentarios tienen una dimensión global y, por lo tanto, preocupan mundialmente.

En suma, no estamos ante una obra construida mediante un único discurso teóricometodológico y articulada por una sola línea argumental, sino ante una compilación que agrupa trabajos de autores/as de ámbitos disciplinares diversos. Aunque, desde luego, el interés y la claridad expositiva se hacen patentes en todos los capítulos, los cuales, si bien obviamente no agotan la amplitud y la complejidad de las temáticas abordadas en ellos, están bien escritos y fundamentados desde el punto de vista teórico, metodológico y empírico, a la vez que todos ellos son pertinentes, de interés y sugerentes. Particularmente, a este respecto, la validez y el mérito del considerable esfuerzo colectivo de alcance global que hay detrás de este libro estriba, sobre todo, en su capacidad de sugerir, en los caminos y posibilidades que abre para futuros trabajos. Estamos, pues, ante un primero y obligado paso en la necesidad de acometer el estudio de la producción de comida, así como de la construcción de los hábitos alimentarios en el crecientemente intervinculado mundo actual, desde perspectivas capaces de integrar la diversidad global y las diferentes disciplinas científicas en ello concernidas.

\section{Referencias bibliográficas}

AA.VV. Seguridad alimentaria y políticas de lucha contra el hambre. Córdoba: Universidad de Córdoba y Oficina de Cooperación Internacional al Desarrollo, 2006.

AA.VV. Derecho a la alimentación y soberanía alimentaria. Córdoba: Universidad de Córdoba y Oficina de Cooperación Internacional al Desarrollo, 2008.

CORBEAU, J.P. Les dimensions cachées de la surcharge pondérale. Sciences Sociales et Santé, 2008, Vol. 26, № 4, p. 113-120.
DE GARINE, I. Introduction. In: BATAILLEBENGUIGUI, M.C. \& COUSON, F. (editors). Cuisines, reflets des sociétés. Paris: Sépia, Musée de l'Homme, 1996.

ESQUINAS ALCÁZAR, J. Biodiversidad y Seguridad. Cuadernos de Estrategia, 2013, № 161, p. 109-156.

GOODY, J. Cuisines, cuisine et classes. Paris: Centre Georges Pompidou, Centre de création industrielle, 1985.

KENNEDY, J.F. Remarks at the Opening Session of the World Food Congress. 1963. Disponible en Internet: http://www.presidency.ucsb.edu/ws/index.php?pid=9249

LÓPEZ GARCÍA, D. Producir alimentos, reproducir comunidad. Redes alimentarias alternativas como formas económicas para la transición social y ecológica. Madrid: Libros en Acción, 2015.

MAULEÓN, J.R. Los canales cortos de comercialización alimentaria como alternativa de los pequeños agricultores ante la globalización: el caso español. En: XXIII Congreso de la Asociación Latino Americana de Sociología. Comisión de trabajo 21, Crisis agropecuaria, globalización y alternativas campesinas, 2001. Disponible en Internet: http:// www.araba.ehu.es/depso/Documentos $\% 20$ de $\% 20$ interes $\% 20 \% 20$ sobre $\% 20$ Sociologia $\% 20 \mathrm{de} \% 20 \mathrm{a} \% 20$ Alimentacion.htm

SAHLINS, M. Culture in Practice. Selected Essays. New York: Zone Books, 2000.

SERRA-MALLOL, CH. Entre local et global: I'alimentation polynésienne: le cas de Tahiti et de Rapa. Anthropologie et Sociétés, 2013, Vol. $37, N^{\circ} 2$, p. 137-153.

SUMPSI VIÑAS, J.M. La volatilidad de los mercados agrarios y la crisis alimentaria mundial, Cuadernos de Estrategia. 2013, № 161, p. 157-187. 

